\title{
OPEN The relationship
} \section{between resting-state functional connectivity, antidepressant discontinuation and depression relapse}

Isabel M. Berwian ${ }^{1,2,3 凶}$, Julia G. Wenzel ${ }^{4}$, Leonie Kuehn ${ }^{4}$, Inga Schnuerer ${ }^{1}$, Lars Kasper ${ }^{1,5}$, Ilya M. Veer ${ }^{4}$, Erich Seifritz ${ }^{2}$, Klaas E. Stephan ${ }^{1,6,7}$, Henrik Walter ${ }^{4,10}$ \& Quentin J. M. Huys $(1)$ $1,2,8,9,10$

The risk of relapsing into depression after stopping antidepressants is high, but no established predictors exist. Resting-state functional magnetic resonance imaging (rsfMRI) measures may help predict relapse and identify the mechanisms by which relapses occur. rsfMRI data were acquired from healthy controls and from patients with remitted major depressive disorder on antidepressants. Patients were assessed a second time either before or after discontinuation of the antidepressant, and followed up for six months to assess relapse. A seed-based functional connectivity analysis was conducted focusing on the left subgenual anterior cingulate cortex and left posterior cingulate cortex. Seeds in the amygdala and dorsolateral prefrontal cortex were explored. 44 healthy controls (age: 33.8 (10.5), 73\% female) and 84 patients (age: 34.23 (10.8), 80\% female) were included in the analysis. 29 patients went on to relapse and 38 remained well. The seed-based analysis showed that discontinuation resulted in an increased functional connectivity between the right dorsolateral prefrontal cortex and the parietal cortex in non-relapsers. In an exploratory analysis, this functional connectivity predicted relapse risk with a balanced accuracy of 0.86 . Further seed-based analyses, however, failed to reveal differences in functional connectivity between patients and controls, between relapsers and non-relapsers before discontinuation and changes due to discontinuation independent of relapse. In conclusion, changes in the connectivity between the dorsolateral prefrontal cortex and the posterior default mode network were associated with and predictive of relapse after open-label antidepressant discontinuation. This finding requires replication in a larger dataset.

A subset of those suffering from Major Depressive Disorder (MDD) achieve remission with antidepressant medication $(\mathrm{ADM})^{1}$. However, this does not imply that the illness is cured as one in three of those achieving remission experience a relapse within six months after ADM discontinuation ${ }^{2}$. Indeed, the burden of depressive illness is in no small part due to their chronic nature with frequent relapses over the lifetime ${ }^{3,4}$. Hence, the management of relapses is of paramount importance. In this situation, predictors of relapse risk are urgently needed to guide decision-making at key decision points such as the discontinuation of ADM after remission has been achieved.

Unfortunately, standard demographic and clinical variables appear to have very limited predictive power ${ }^{5}$, requiring attention to be turned to more complex measurements. Here, we examined resting-state functional connectivity-a complex yet clinically feasible neuroimaging procedure ${ }^{6}$.

\footnotetext{
${ }^{1}$ Translational Neuromodeling Unit, University of Zurich and ETH Zurich, Zurich, Switzerland. ${ }^{2}$ Hospital of Psychiatry, University of Zurich, Zurich, Switzerland. ${ }^{3}$ Princeton Neurosciene Institute, Princeton University, Princeton, USA. ${ }^{4}$ Charité Universitätsmedizin, Campus Charité Mitte, Berlin, Germany. ${ }^{5}$ Institute of Biomedical Engineering, ETH Zurich and University of Zurich, Zurich, Switzerland. 'Wellcome Trust Centre for Neuroimaging, University College London, London, UK. ${ }^{7}$ Max Planck Institute for Metabolism Research, Cologne, Germany. ${ }^{8}$ Division of Psychiatry and Max Planck Centre for Computational Psychiatry and Ageing Research, University College London, London, UK. ${ }^{9}$ Camden and Islington NHS Foundation Trust, London, UK. ${ }^{10}$ These authors contributed equally: Henrik Walter and Quentin J. M. Huys. ${ }^{\circledR}$ email: iberwian@princeton.edu
} 
Indeed, a substantial body of research has examined resting-state functional connectivity (RSFC) in the context of depression. Abnormal RSFC in MDD has been observed in three core networks, i.e. the so-called "default mode network" (DMN), the central executive network (CEN) and the salience network (SN). Specifically, in $\mathrm{MDD}$, the connectivity within the anterior and posterior DMN is increased and connectivity between the anterior $D M N$ and the affective and salience network is also altered ${ }^{7,8}$. However, a reduction in connectivity within the $\mathrm{DMN}$ has also been reported amongst medicated patients and those with recurrent illness ${ }^{9}$. Connectivity within the CEN and SN and connectivity to the posterior DMN are reduced ${ }^{7,8}$.

In addition to abnormal RSFC in current depression ${ }^{10-12}$, the subgenual anterior cingulate cortex (sgACC) appears to have a particularly central role in relation to treatment response, remitted depression and relapse. First, neuroimaging measures of metabolic activity within this region have been shown to have predictive power for response to antidepressant and other treatments ${ }^{13,14}$. Second, connectivity changes in these regions have also been observed in remitted patients off medication and in populations with correlates of depression such as trauma, childhood maltreatment, subclinical depression and familial risk in children. These include increased connectivity between the sgACC and both the medial prefrontal cortex (mPFC) and the posterior cingulate cortex (PCC), as well as bidirectional changes in connectivity between the sgACC and the dorsolateral PFC (dlPFC), the amygdala and the hippocampus $\left({ }^{15-24}\right.$; though see also $\left.{ }^{25}\right)$. Third, and most importantly, decreased interhemispheric sgACC connectivity from the left to the right has been suggested as a marker of resilience to relapse in patients off medication ${ }^{26}$, while increased connectivity between the sgACC and regions in the CEN differentiates patients who will experience a relapse and those who will not ${ }^{27}$.

The results of studies examining the impact of ADM initiation on connectivity are heterogeneous ${ }^{28-39}$. One of the more replicated findings is a decrease in connectivity between PCC and various brain regions (including amygdala $^{31}$, right inferior temporal gyrus ${ }^{36}$, right inferior frontal gyrus ${ }^{33}$ ), suggesting a normalisation of increased PCC connectivity with these regions in comparison to the prior, medication-free, state. Increases between the PCC and the ACC and mPFC have been reported in patients who receive treatment, but do not remit ${ }^{32}$.

Outstanding gaps in the literature include characterization of RSFC in the remitted medicated state, difference in RSFC between patients who relapse and remain well after antidepressant discontinuation and the neurobiological effects underpinning discontinuation. We aimed to address these gaps in the context of the AIDA study-a two-centre observational randomized study that followed patients as they discontinued their ADM for six months. The fundamental goal of the study was to identify biomarkers of relapse risk and uncover neurobiological changes due to discontinuation. To this end, we examined (1) whether RSFC connectivity differs between remitted medicated patients and controls; (2) whether pre-discontinuation RSFC differs between patients who relapse after discontinuation and those who remain well; (3) the effects of discontinuation on RSFC; and (4) to what extent changes in RSFC due to discontinuation are related to subsequent relapses. Based on the reviewed literature, we focused on the sgACC as seed region to address questions 1 and 2 and on the PCC for questions 3 and 4 .

\section{Methods and materials}

Study design. The AIDA study design is depicted in Fig. 1. Briefly, we focused on remitted patients on ADM who wanted to discontinue their medication. Participants were randomised to one of the two study arms. In group MA1-D-MA2, participants underwent the first main assessment (MA1), then gradually discontinued their medication over up to 18 weeks, and then underwent a second main assessment (MA2). In group MA1-MA2-D, participants underwent two main assessments and then discontinued. Hence, the group name indicates the order of events and "D" indicates discontinuation.

After discontinuation, all patients were contacted by telephone at weeks 1, 2, 4, 6, 8, 12, 16 and 21 to assess relapse status. If subjects exhibited signs of a relapse, a in-person structured clinical interview was performed $\left(\mathrm{SCID}-\mathrm{I}^{40}\right)$ to establish the presence of a DSM-IV-TR major depressive episode. If this was present, participants underwent a final assessment (FA) at that point, otherwise the final assessment took place in week 26. Healthy controls (HC) matched for age, sex and education were only assessed once (MA1).

All patients underwent a resting-state functional magnetic resonance imaging (rsfMRI), self- and observerrated reports during each main assessment (MA1 and MA2) and HC once at MA1. See supplementary section S1.2 for observer-rated and self-report measures. Participants were recruited between July 2015 and January 2018 .

Participants. The AIDA study recruited participants who had experienced one severe ${ }^{41}$ or multiple depressive episodes; had initiated antidepressant treatment during the last depressive episode and now achieved stable remission; and had reached the decision to discontinue their medication independently from and prior to study participation. Detailed inclusion and exclusion criteria are listed in the supplementary section S1.1. All participants gave informed written consent and received monetary compensation for the time of participation. Ethical approval for the study was obtained from the cantonal ethics commission Zurich (BASEC: PB_2016-0.01032; KEK-ZH: 2014-0355) and the ethics commission at the Campus Charité-Mitte (EA 1/142/14). All experiments were performed in accordance with relevant guidelines and regulations.

Seed region selection. Seven seeds of interest (Fig. 2a) were identified based on existing literature.The sgACC seeds with MNI coordinates $\pm 4,21,-8$ were chosen as they had been used in several previous studies with patients with remitted MDD $\mathrm{MD}^{15,19-21,27}$ and clearly mapped on the anatomical region of the sgACC during visual inspection. For the left PCC we chose the MNI coordinates - 3, - 39, 39 as identified by Fox and colleagues $^{42}$ as a central part of the task-negative network and were reported to have normalised RSFC after $\mathrm{ADM}_{\text {initiation }}{ }^{35}$. dlPFC coordinates $(\mathrm{MNI} \pm 37,26,31)$ were taken from Sheline and colleagues ${ }^{43}$ and have been 
Discontinuation $\mathrm{x}$ relapse interaction

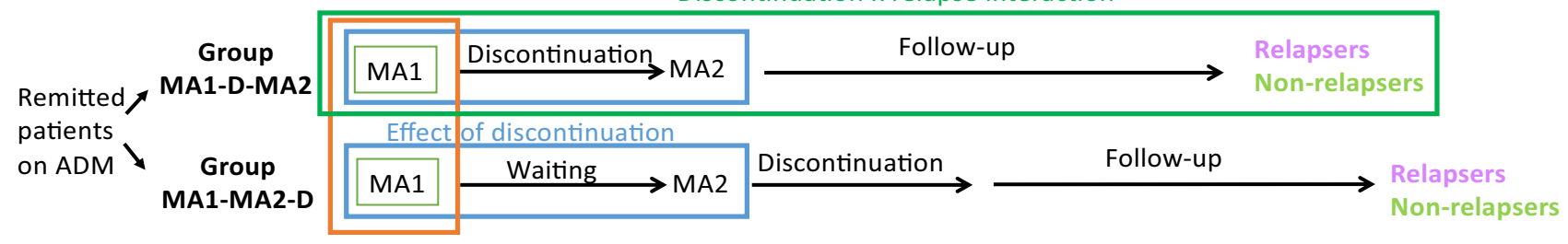

Matched

Healthy

Patients vs healthy controls

Controls

Figure 1. Study Design: Remitted patients on antidepressant medication (ADM) and matched healthy controls were included in the study and assessed during main assessment 1 (MA1). Patients were randomised to groups MA1-D-MA2 or MA1-MA2-D, where the name indicates the order of events and "D" indicates discontinuation. In group MA1-D-MA2, they underwent MA1, then discontinued their medication followed by main assessment 2 (MA2). In group MA1-MA2-D, they underwent MA1 and MA2 first and only discontinued thereafter. After discontinuation, all patients were followed up for six months to ascertain relapses. Comparison of patient and control groups at MA1 was used to examine the remitted medicated state cross-sectionally (orange). Data collected at MA1 from all patients who subsequently completed the study as relapsers or non-relapsers, i.e. relapsers and non-relapsers from both groups combined were examined to examine associations with relapse. The interaction between time point (MA1 and MA2) with group (MA1-D-MA2 and MA1-MA2-D) examined the impact of discontinuation (blue). Finally, the interaction between time point (MA1 and MA2) and relapse in group MA1-D-MA2 was used to examine changes due to discontinuation that led to relapse or resilience (dark green).

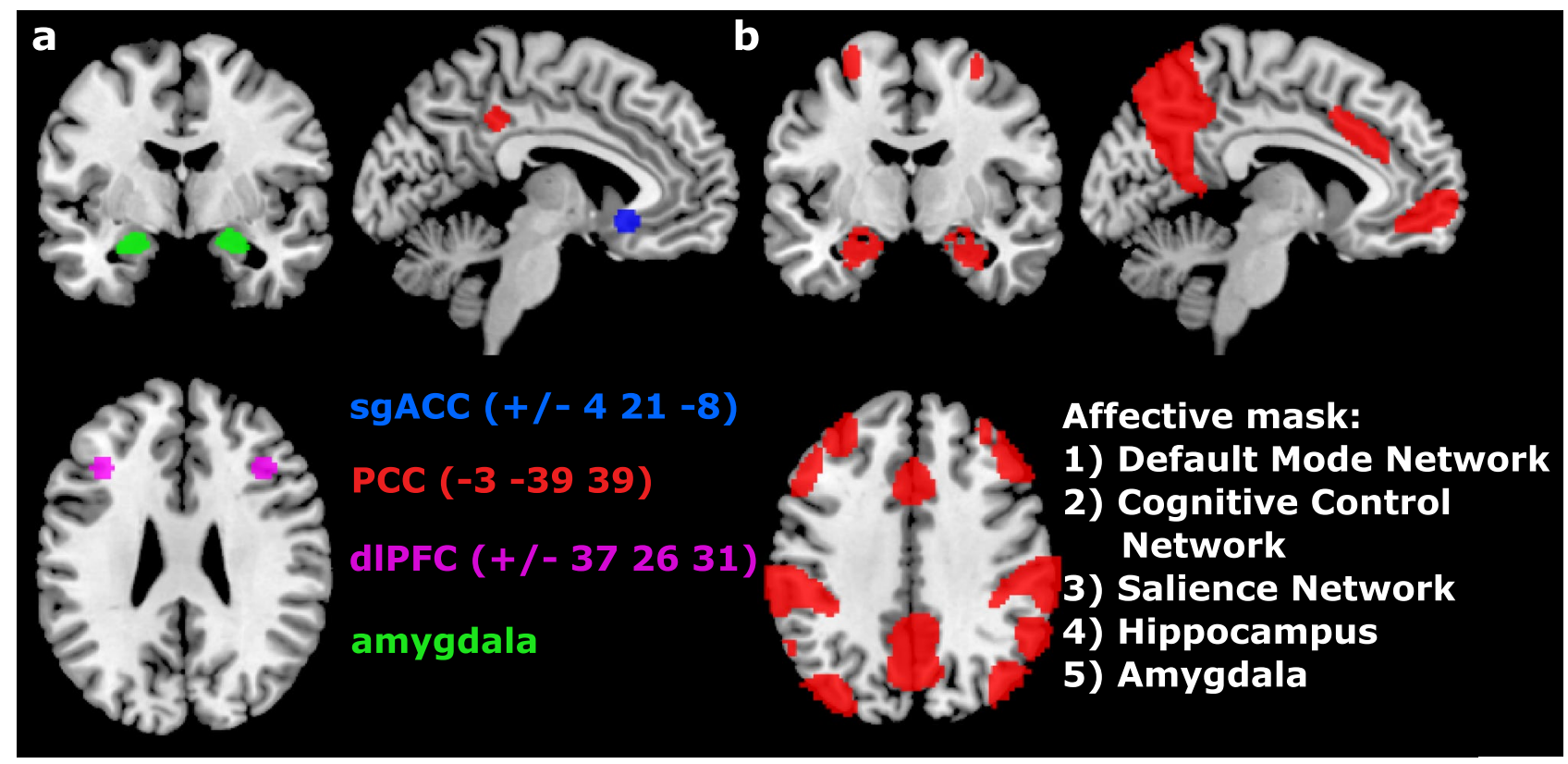

Figure 2. Seeds and Mask: Depicted are the seeds (a) used in the analysis and the affective mask (b) containing all voxels of interest. sgACC subgenual anterior cingulate cortex, $P C C$ posterior cingulate cortex, $d l P F C$ dorsolateral prefrontal cortex.

used widely in the literature. Unless stated otherwise, seeds were created by putting a $6 \mathrm{~mm}$ sphere around the coordinates. Amygdala seeds were extracted from the Harvard-Oxford Subcortical Probability Atlas by including all voxels with at least $80 \%$ probability of being in the amygdala. For each analysis, one seed was designated a priori as the main seed of interest and the other six as exploratory seeds.

Resting-state fMRI analysis. Image acquisition and preprocessing. The sequences used for image acquisition are described in the supplementary section S1.4. Imaging data was preprocessed with a FSL (FMRIB Software Library v5.0) pipeline detailed in supplementary section S1.5.

First-level analyses. First level analyses were conducted with the CONN toolbox ${ }^{44}$. We included motion regressors (see section motion correction for details), 10 regressors computed using aCompCor in the CONN toolbox 
(5 principal components of white matter and cerebrospinal fluid signals each), and bandpass filtered the data to retain frequencies between 0.01 and $0.1 \mathrm{~Hz}$. All covariates were also regressed out of the seed regions. Then, the average signal of all voxels within subject-specific grey matter masks of each seed was computed and correlated with all voxels in the brain. Resulting correlation coefficients were z-transformed for second-level analyses.

Analyses to control for effects of motion and study site are described in detail in supplementary section S1.6 and S1.7. Subjects with a framewise displacement (FD) of $1 \mathrm{~mm}$ from one volume to the next and with a mean SNR smaller than 30 were excluded from further analyses.

Second-level analyses. Second-level analyses were conducted with SPM12 (version: v7219). For all analyses with our chosen main seed, we report peak-level results family-wise error (FWE) corrected at the voxel level at 0.05 and depict the respective clusters in figures. Altered RSFC of the selected seeds in acute and remitted depression has mainly been reported for voxels which are part of the DMN, the CEN, the SN, the amygdala and the hippocampus $7,8,15,17,19-21,24$. As FWE-corrected results depend on the search volume of the analysis, we constructed an "affective mask" containing all voxels within the DMN, the CEN and the SN, as well as voxels within anatomical masks of the bilateral amygdalae and the hippocampi (Fig. 2b) as these areas show abnormal RSFC in affective disorders. Details on included areas in the mask can be found in supplementary section S1.8 Exploratory analyses were considered significant after Bonferroni correction for all six seeds at $p=0.0083$.

Our first analyses focused on the first assessment MA1. To identify abnormalities persisting into the remitted medicated state we compared RSFC between the left sgACC and the affective mask between patients and HC using an independent-sample $t$-test. To identify markers of prospective relapse, we repeated this analysis comparing patients who did and did not go on to relapse. Exploratory analyses examined the RSFC between the affective mask and the right sgACC, the left PCC, right and left amygdala and right and left dlPFC seed regions.

Next, we examined the impact of discontinuation. To test the hypothesis that RSFC between the PCC and the affective mask would increase due to discontinuation, we applied a mixed-design analysis of variance (ANOVA) with group (MA1-D-MA2 and MA1-MA2-D) as between-subject factor and time point as within-subject factor (MA1 and MA2). Where significant interaction effects were found, we conducted the following post-hoc tests: paired sample $t$-test in group MA1-D-MA2 to identify changes related to discontinuation; paired sample $t$-test in group MA1-MA2-D to investigate test-retest reliability and an independent sample $t$-test between groups MA1-D-MA2 and MA1-MA2-D at MA1 to verify that no random allocation differences occurred prior to discontinuation. We repeated this analyses using the right and left sgACC, right and left amygdala and right and left dlPFC as seeds for exploratory analyses.

Finally, we attempted to gain insight into how the effects of discontinuation might relate to relapse by examining the interaction of discontinuation and relapse. For this, we only included patients from group MA1-D-MA2 and applied a mixed-design ANOVA with time point as within-subject factor (MA1 and MA2) and relapse status (relapsers and non-relapsers) as between subject factor. Post-hoc paired sample $t$-tests were performed within the relapse group to identify changes related to relapse and paired $t$-tests in the no-relapse group to identify changes related to resilience.

Additional sanity checks and exploratory analyses are described in supplementary section S1.9.

Covariates. RSFC that were found to differ significantly between or within groups were correlated with several covariates including age, gender and site as covariates of no interest and rumination score, residual depression score, medication load and disease severity. Computation of medication load and severity factor is outlined in supplementary section S1.3.

Post-hoc prediction analyses. As suggested by a reviewer we examined whether associations between RSFC and relapse could predict relapse risk out-of-sample. To this end, we fitted logistic regressions nested in a leaveone-out cross-validation for the main significant cluster in the interaction analyses that indicated differences in changes in RSFC between MA1 und MA2 in relapsers and non-relapsers. Group membership of the left-out participant was predicted using parameter estimates (regression weights) obtained from the participants included in the fit. Finally, the balanced accuracy for predicted group memberships was computed.

\section{Results}

Participants. The consort diagrams (Figure S1 and Figure S2) show numbers of patients and controls at each study stage and reasons for dropouts. After exclusion, 84 patients (mean [SD] age, 34.23 (10.8) years, 17 men [20\%]) and 44 controls (mean [SD] age, 33.8 (10.5) years, 12 men [27\%]) were included at MA1. 61 (73\%) patients and $27(61 \%)$ controls were assessed in Zurich, the remaining participants in Berlin. Further details on demographic and clinical characteristics are displayed in Table 1. Patients scored higher on the rumination brooding subscale. This was particularly accentuated in patients who went on to relapse. $69 \%$ of our sample took a selective serotonin reuptake inhibitor, $27 \%$ a serotonin-norepinephrine reuptake inhibtor and $4 \%$ an antidepressant from a different class.

Motion and site effects. Comparing participants with high and low motion during MA1 yielded no significant difference between these groups for any of the seeds. Hence, we decided to only exclude participants with FD $>1$ between two volumes but added no additional motion regressors and did not censor any scans with stick regressors.

There was an effect of site on the temporal signal-to-noise ratio $(t(126)=7.76, p<0.001$, Figure S3). To examine if this impacted significant effects, we correlate the significant RSFC with site as covariate of no interest. 


\begin{tabular}{|c|c|c|c|c|c|c|}
\hline \multirow[b]{2}{*}{ Variable $^{\mathrm{a}}$} & \multicolumn{3}{|c|}{ Patients vs. healthy controls } & \multicolumn{3}{|c|}{ Relapsers vs. non-relapsers } \\
\hline & Patients $(n=84)$ & $\begin{array}{l}\text { Healthy controls } \\
(\mathrm{n}=44)\end{array}$ & $p$ value & Relapsers $(n=29)$ & Non-relapsers $(n=38)$ & $p$ value \\
\hline \multicolumn{7}{|l|}{ Demographics } \\
\hline Age & $34.23(10.8)$ & $33.8(10.5)$ & 0.83 & $36.76(11.1)$ & $31.79(10.3)$ & 0.062 \\
\hline Male sex, No. (\%) & $17(20)$ & $12(27)$ & 0.37 & $7(24)$ & $6(16)$ & 0.39 \\
\hline Site Zürich, No. (\%) & $61(73)$ & $27(61)$ & 0.19 & $20(69)$ & $28(74)$ & 0.67 \\
\hline \multicolumn{7}{|l|}{ Clinical measures } \\
\hline $\begin{array}{l}\text { Number of prior } \\
\text { episodes }\end{array}$ & - & - & - & $2.76(1.8)$ & $2.32(1.6)$ & 0.29 \\
\hline Residual depression ${ }^{\mathrm{b}}$ & $3.38(3.7)$ & $0.70(1.1)$ & $<0.001$ & $3.52(5.14)$ & $2.87(2.3)$ & 0.49 \\
\hline Disease severity $^{c}$ & - & - & - & $0.08(0.4)$ & $-0.05(0.33)$ & 0.14 \\
\hline Medication load $^{c}$ & - & - & - & $0.007(0.004)$ & $0.008(0.004)$ & 0.33 \\
\hline Month of ADM intake $^{\mathrm{d}}$ & - & - & - & 24 & 28 & 0.77 \\
\hline Psychotherapy $^{c}$ & - & - & - & $0.40(0.39)$ & $0.39(0.39)$ & 1 \\
\hline Comorbidities $^{\mathrm{b}}$ & - & - & - & $0.72(1.03)$ & $0.61(1.03)$ & 0.64 \\
\hline Days of tapering & - & - & - & $52.86(40.18)$ & $51.53(43.74)$ & 0.90 \\
\hline \multicolumn{7}{|l|}{ Covariates of interest } \\
\hline Brooding $^{\mathrm{b}}$ & $9.65(2.6)$ & $8.14(2.3)$ & 0.001 & $10.55(2.77)$ & $8.71(1.9)$ & 0.002 \\
\hline \multicolumn{7}{|l|}{ Imaging measures } \\
\hline Framewise displacement & $0.11(0.05)$ & $0.10(0.05)$ & 0.42 & $0.12(0.07)$ & $0.11(0.04)$ & 0.37 \\
\hline
\end{tabular}

Table 1. Participant characteristics. ${ }^{a}$ Unless stated otherwise, mean (SD) are shown. ${ }^{b}$ Determined as follows: residual depression: Inventory of Depressive Symptomatology-Clinician Rated ${ }^{45}$; brooding: brooding subscale of the German version of the Response Style Questionnaire ${ }^{46}$; comorbidities: number of past and present psychiatric diagnoses. ${ }^{\mathrm{c}}$ Computation of the variables is described in the supplementary section S1.3. ${ }^{\mathrm{d}}$ Median is provided and a non-parametric Wilcoxon rank sum test was applied to test significance.

Overall functional connectivity patterns. As a sanity check we computed the connectivity of all left seeds (left sgACC, left PCC, left dlPFC and left amygdala) with all voxel in the affective mask (including voxels from the DMN, the CEN, the SN, the amygdala and the hippocampus) across all participants at MA1. The connectivity pattern replicated the patterns established in the literature (Figure S4).

The remitted and medicated state. To identify abnormal RSFC in the remitted medicated state, we compared remitted patients on ADM and controls. For this analysis, we focused on connectivity between the left sgACC, i.e. the main a-priori seed, with voxels in the affective mask. RSFC between the seed with voxels in the affective mask did not differ between remitted patients on ADM and controls. No differences were found for any of the exploratory seeds (right sgACC, left PCC, right and left dlPFC and right and left amygdala).

Relapse effects. The left sgACC was again designated as the main a-priori seed to examine associations of RSFC with relapse. RSFC between the left sgACC and voxels in the affective mask at MA1 did not differ between patients who did and did not go on to relapse during the follow-up period. No differences were found for any of the exploratory seeds.

Discontinuation effects. The left PCC was designated as the main a-priori seed to examine effects of discontinuation. RSFC of the left PCC with voxels in the affective mask did not show an interaction between time point of assessment (MA1 or MA2) and discontinuation group (MA1-D-MA2 and MA1-MA2-D). No interaction effects were found for any of the exploratory seeds.

Interaction of discontinuation and relapse. To examine the interaction between changes in RSFC before and after discontinuation and subsequent relapse, we first focused on the changes in the connectivity of the left PCC. These analysis was conducted in group MA1D-MA2 only. No changes were identified for RSFC of the left PCC. However, examining the exploratory seeds a significant interaction between discontinuation and relapse emerged for RSFC between the right dlPFC and two peaks in the right posterior DMN, namely in the parietal cortex (Fig. 3a, b; Table 2) and the posterior cingulate cortex (Fig. 3a, d). Post-hoc tests did not reveal differences between relapsers and non-relapsers before discontinuation. The significant interaction was driven by an increase in RSFC in patients who discontinued but did not go on to relapse: RSFC between the right dlPFC and the right parietal cortex increased with discontinuation in those who remained well (Fig. 3a, c; note the slight shift in the parietal cortex for the post-hoc test depicted in blue), while it decreased numerically without reaching statistical significance in those who went on to relapse. None of the post-hoc tests on the connectivity between right dIPFC and PCC reached significance, although a similar pattern of results is visible as for the connectivity of right dlPFC and parietal cortex (compare Fig. 3b, d). The effects did not remain significant when including participants with more than $1 \mathrm{~mm}$ FD and censuring the scans with stick regressors. 

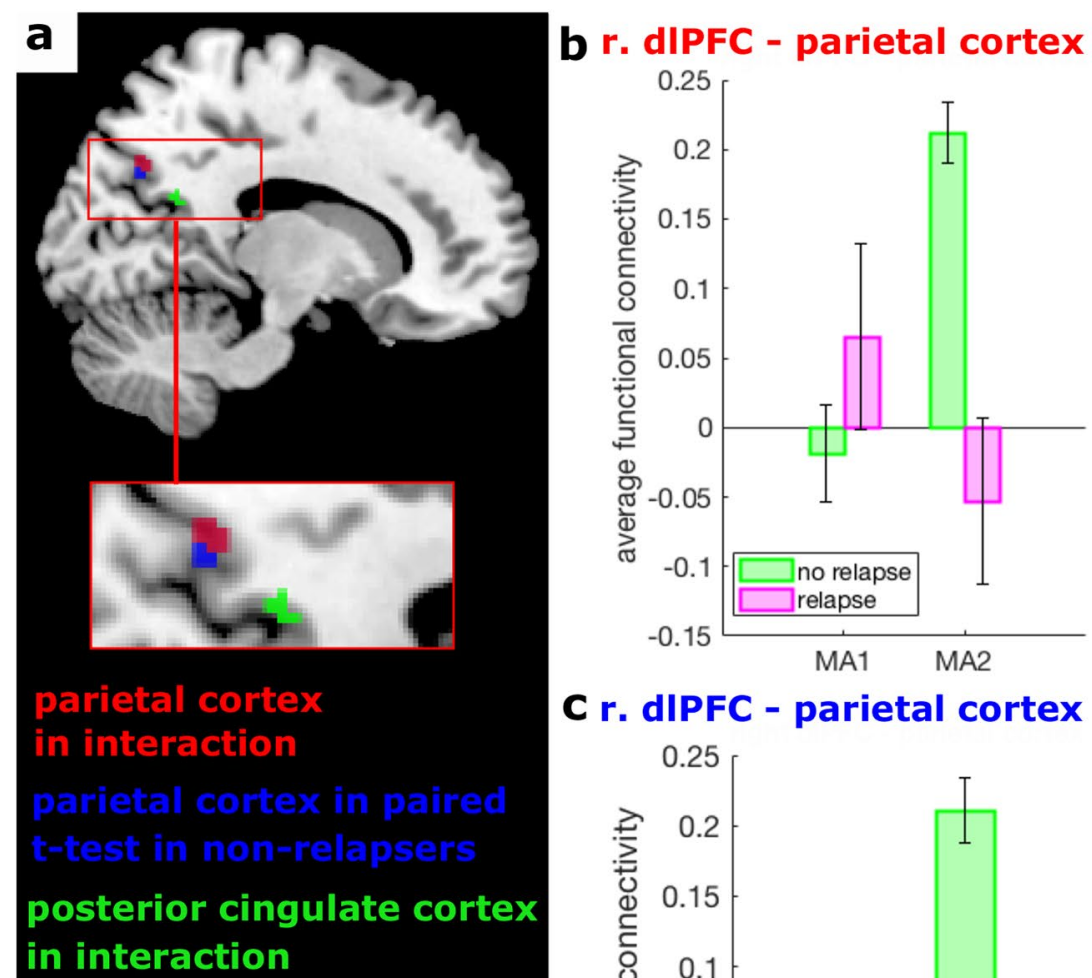

\section{C r. dIPFC - parietal cortex}

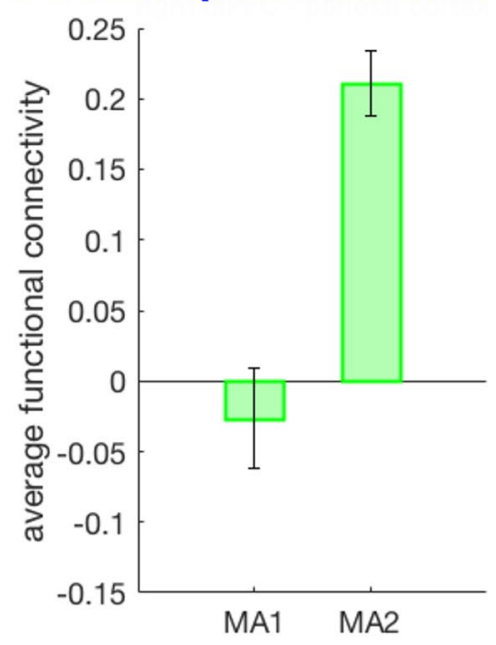

d

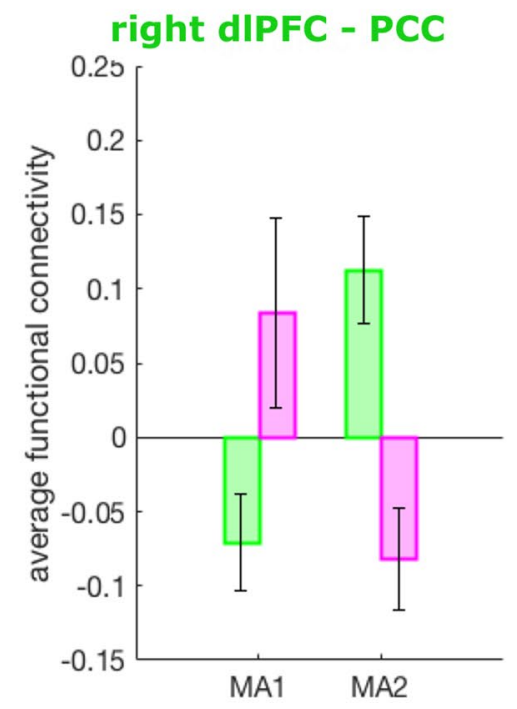

Figure 3. Discontinuation relapse interaction effect: (a) Depicted are clusters for significant interactions between discontinuation and relapse between our choosen seed in the right dorsolateral prefrontal cortex (dlPFC, magenta) and the parietal cortex (red) and the posterior cingulate cortex (PCC, green) and a paired $t$-test in non-relapsers only for RSFC between right dlPFC and the parietal cortex (blue). (b-d) The Fisher z-transformed average functional connectivity between the right dlPFC and the surviving clusters in the parietal cortex and the PCC is depicted for the interaction between relapsers and non-relapsers (b, d, respectively). Specifically, depicted are significant interaction effects with $p<0.0083$ which survive our threshold corrected for multiple comparison. Exact $p$ values are given in Table 2. (c) The paired post-hoc $t$-test in nonrelapsers for the interaction effect in relation to the parietal cortex shown in (b). Error bars indicate standard errors. 


\begin{tabular}{|c|c|c|c|c|c|c|c|c|c|}
\hline \multirow[b]{2}{*}{ Contrast } & \multirow[b]{2}{*}{ Region (BA) } & \multicolumn{3}{|c|}{$\begin{array}{l}\text { Peak MNI } \\
\text { coordinates }\end{array}$} & \multirow[b]{2}{*}{$\mathbf{k}$} & \multirow[b]{2}{*}{$p$ (cluster) } & \multirow[b]{2}{*}{ T-value } & \multirow[b]{2}{*}{$\mathbf{Z}$} & \multirow[b]{2}{*}{$p$ (peak) } \\
\hline & & $\mathbf{x}$ & $y$ & $\mathbf{z}$ & & & & & \\
\hline \multirow{2}{*}{ Interaction in F-test } & Parietal cortex (BA 7) & 14 & -66 & 38 & 30 & 0.002 & 5.05 & 5.21 & 0.001 \\
\hline & PCC (BA 23) & 12 & -54 & 14 & 18 & 0.006 & 5.06 & 5.2 & 0.001 \\
\hline Paired $T$-test in non-relapsers & Parietal cortex (BA 7) & 14 & -66 & 36 & 30 & $<0.001$ & 8.2 & 5.43 & 0.001 \\
\hline
\end{tabular}

Table 2. Significant discontinuation relapse effects for the dorsolateral prefrontal cortex. BA Brodmann area, MNI Montreal Neurological Institue, PCC posterior cingulate cortex.

Post-hoc prediction analyses. Using the average functional connectivity between the right dlPFC and the cluster in the parietal cortex (shown in red in Fig. 3a) at MA2, relapse could be predicted out-of-sample with a balanced accuracy of 0.86 (Figure S5). Similarly, using the change in that RSFC from MA1 to MA2 also predicted relapse with a balanced accuracy of 0.86 . Note that the data used in the second prediction analysis is not orthogonal to the significant interaction effect and, hence, does not provide independent evidence for the prediction.

Exploratory analyses without correction for multiple comparisons. No significant effects emerged for any of the exploratory seeds in any of the analyses when we dropped the correction for multiple comparison for number of seeds and set the required significance level to 0.05 FWE-corrected. In Table S1, we additionally report results for RSFC for our main seeds for all analyses at an uncorrected significance level of 0.001 .

Covariates. We correlated RSFC between the right dlPFC and the cluster in the parietal cortex in nonrelapsers before they discontinued as well as the change accompanying the discontinuation with our pre-specified covariates, but found no significant correlations.

\section{Discussion}

We examined resting-state functional connectivity in the context of antidepressant discontinuation in stable remission from Major Depressive Disorder. We found a significant interaction of discontinuation and relapse in the subgroup of patients who were tested before and after discontinuation. This interaction was driven by those who remained well during the follow-up period showing a significant increase in RSFC between the dlPFC and two areas in the posterior DMN, namely the parietal cortex and the PCC, whereas patients who went on to relapse did not show this increase and instead showed a numerical decrease that did not reach statistical significance. Both dlPFC-parietal cortex RSFC after discontinuation and the change in RSFC were predictive of relapse in post-hoc exploratory leave-one-out cross-validation analyses.

There are several notable negative results. First, we found no difference between remitted, medicated patients and matched controls. Second, patients who went on to relapse after ADM discontinuation did not differ from those who remained well. Third, discontinuation did not result in detectable changes in RSFC between the PCC and voxels within our affective mask, nor any of the exploratory seeds.

Our data suggests that an increase in RSFC between the right dlPFC and the posterior DMN, in particular the parietal cortex, relates to resilience to relapse after ADM discontinuation. An effect of this kind was expected a priori as it reflects prior findings showing a decrease in PCC connectivity with ADM initiation ${ }^{33,36,31}$. However, unlike previous reports of an increase in the RSFC of the CEN in those who go on to relapse ${ }^{27}$, we here observed an increase in right dlPFC-parietal connectivity in patients who remained well. These findings hence point towards a compensatory effect in response to discontinuation that might protect patients from relapse. Whilst the default mode has been related to rumination, the right dlPFC has been extensively related to regulation ${ }^{47}$ and conceptual-emotional information integration ${ }^{48}$. It is hence tempting to speculate that the increase in connectivity might reflect emotion regulation strategies and rumination, both of which influence the course of depression ${ }^{49,50}$.

Exploratory post-hoc analyses indicated that RSFC between the right dlPFC and the significant cluster in the parietal cortex can predict relapse risk above chance for new, previously unseen patients. The analysis suggests that the connectivity effect may not only be associated with relapse, but may also have some predictive potential. In fact, the measure of RSFC after discontinuation is sufficient to predict subsequent relapse, indicating that one scanning session may be sufficient. Being able to predict relapse risk for new patients is crucial for translation to the clinical setting, where clinicians need to make treatment decisions using models that were developed without the patient whose treatment course they need to decide upon. However, the present result also needs to be interpreted with great caution. First, the analysis was exploratory and focused on significant clusters. Second, the sample size included in this analysis was very small, which can inflate the result ${ }^{51}$. Hence, the result can be seen as a first hint at a potential predictive marker of relapse risk which, however, requires replication in an independent and larger dataset.

We also emphasize the difficulties inherent in interpreting the absence of an effect, and we note the problems around reliability of RSFC measures for brief scans as in our study ${ }^{52}$. Although these results do not provide evidence for the absence of effects, power considerations can nevertheless inform the interpretation by indicating the size of effects a study of this size should have identified. Two-tailed two-sample $t$-tests comparing 84 patients to 44 healthy controls and 29 relapsers to 38 non-relapsers have a power of 0.8 to detect medium $(\mathrm{d}=0.52)$ and large $(d=0.70)$ group differences, respectively, and a power of 0.95 to detect effects of $d=0.67$ and 0.90 , respectively 
(using $\mathrm{G}^{\star}$ Power $3.1^{53}$ ). As such, large effects above 0.5 are unlikely, and hence we judge it unlikely that restingstate connectivity revealed with a seed-based approach based on short scans as in this study have clinical utility ${ }^{54}$.

The lack of replications of previous results could relate to specific difference in the sample or due to methodological limitations. At the present stage, it is not possible to disentangle these aspects. Hence, we will briefly point out difference in relation to the sample and discuss methodological limitations in the next section.

The absence of significant RSFC differences between remitted MDD patients on ADM and healthy neverdepressed controls contrasts with results in the literature ${ }^{19,24}$. The only major difference between previous and the present study is the medication status: all previous involving remitted patients focused on unmedicated samples ${ }^{15,17,19-21,24}$, while our patient group had been medicated for a median duration of two years.

The absence of differences in RSFC between patients who went on to relapse and patients who remained well after ADM discontinuation is similarly surprising, given that relapse and resilience independent of discontinuation have been reported to be associated with abnormal RSFC of the sgACC ${ }^{26,27}$. We again note that our sample was assessed in the medicated state before discontinuation. The absence of a difference in our study raises the possibility that previous findings relate more broadly to relapse risk, and that our lack of findings speaks only to the specific induction of relapse through discontinuation.

Next, ADM discontinuation did not reveal any effects. This result, again, is unexpected given that ADM initiation has been shown to induce changes in the connectivity of the PCC ${ }^{31,33,36}$. One potential explanation could be that the timing of the second measurement five half-lives after ADM discontinuation was too early to see effects.

This would in turn suggest a normalisation of RSFC lasting into the unmedicated state.

Limitations and strengths. The study has both strength and limitations. Amongst its strengths is that, to our knowledge, this is the first study examining neurobiological effects in the context of ADM discontinuation and relapse thereafter. The lack of findings and replications in our study, however, might result from some of its limitations. First, the resting-state scan was only $5.5 \mathrm{~min}$, which is relatively short. Second, the evidence in favour of abnormal RSFC of the sgACC, on which we based our hypotheses and analyses, is admittedly heterogeneous and also does rely on partially overlapping samples ${ }^{15,19-21}$. Third, the signal-to-noise ratio differed between the study sites, potentially reducing the power to find effects in the bigger sample from Zurich. Fourth, the fact that patients were on medication from several classes might have increased the heterogeneity of the medications' influence on RSFC and in that it reduced the power to identify significant effects. Fifth, no standard approach for motion correction exists. Hence, cut-off choices are arbitrary. To avoid spurious correlation due to motion ${ }^{55,56}$, we choose a priori a more conservative criterion by excluding all participants with more than $1 \mathrm{~mm}$ FD. Sixth, the naturalistic design does not allow us to disentangle pharmacological effects of discontinuation from the potential psychological effect of knowing that the ADM is discontinued. Seventh, the sample size in particular for relapse effect was rather small. Nevertheless, as discussed above, it should be sufficient to detect moderate effects, which we did not find. Finally, these analyses followed previous studies of RSFC in depression, which primarily used a seed-based approach. More advanced analyses could yet reveal meaningful changes.

\section{Conclusion}

We found no RSFC differences between remitted, medicated patients and healthy controls, between relapsers and non-relapsers or as a consequence of discontinuation. This raises the possibility-but emphatically does not show - that antidepressants normalise RSFC and that this normalisation persists at least for a brief period into the unmedicated state. We did, however, find tentative evidence for a potential marker of resilience to relapse after $\mathrm{ADM}$ discontinuation in terms of an increased connectivity between the right dlPFC and the posterior DMN.

\section{Data availability}

The datasets generated and analysed during the current study are not publicly available due to constrictions by the ethical rules to but are available from the corresponding author on reasonable request and in line with the ethical rules.

Received: 19 June 2020; Accepted: 26 November 2020

Published online: 18 December 2020

\section{References}

1. Rush, A. J. et al. Bupropion-SR, sertraline, or venlafaxine-XR after failure of SSRIs for depression. N. Engl. J. Med. 354, 1231-1242 (2006).

2. Geddes, J. R. et al. Relapse prevention with antidepressant drug treatment in depressive disorders: a systematic review. Lancet 361, 653-661 (2003).

3. Angst, J., Gamma, A., Sellaro, R., Lavori, P. W. \& Zhang, H. Recurrence of bipolar disorders and major depression. A life-long perspective. Eur. Arch. Psychiatry Clin. Neurosci. 253, 236-240. https://doi.org/10.1007/s00406-003-0437-2 (2003).

4. Lépine, J.-P. \& Briley, M. The increasing burden of depression. Neuropsychiatr. Dis. Treat. 7, 3-7 (2011).

5. Berwian, I. M., Walter, H., Seifritz, E. \& Huys, Q. J. Predicting relapse after antidepressant discontinuation a systematic review. Psychol. Med. 47, 426-437 (2017).

6. O'Connor, E. E. \& Zeffiro, T. A. Why is clinical fmri in a resting state?. Front. Neurol. 10, 420 (2019).

7. Mulders, P. C., van Eijndhoven, P. F., Schene, A. H., Beckmann, C. F. \& Tendolkar, I. Resting-state functional connectivity in major depressive disorder: a review. Neurosci. Biobehav. Rev. 56, 330-344 (2015).

8. Kaiser, R. H., Andrews-Hanna, J. R., Wager, T. D. \& Pizzagalli, D. A. Large-scale network dysfunction in major depressive disorder: a meta-analysis of resting-state functional connectivity. JAMA Psychiatry 72, 603-611 (2015).

9. Yan, C.-G. et al. Reduced default mode network functional connectivity in patients with recurrent major depressive disorder. Proc. Natl. Acad. Sci. USA 116, 9078-9083 (2019).

10. Dichter, G. S., Gibbs, D. \& Smoski, M. J. A systematic review of relations between resting-state functional-mri and treatment response in major depressive disorder. J. Affect. Disord. 172, 8-17 (2015). 
11. Dunlop, B. W. \& Mayberg, H. S. Neuroimaging-based biomarkers for treatment selection in major depressive disorder. Dialogues Clin. Neurosci. 16, 479-490 (2014).

12. Greicius, M. D. et al. Resting-state functional connectivity in major depression: abnormally increased contributions from subgenual cingulate cortex and thalamus. Biol. Psychiatry 62, 429-437 (2007).

13. Mayberg, H. S. et al. Cingulate function in depression: a potential predictor of treatment response. NeuroReport 8, 1057-1061 (1997).

14. McGrath, C. L. et al. Pretreatment brain states identify likely nonresponse to standard treatments for depression. Biol. Psychiatry 76, 527-535 (2014).

15. Bhaumik, R. et al. Multivariate pattern analysis strategies in detection of remitted major depressive disorder using resting state functional connectivity. Neuroimage Clin. 16, 390-398 (2017).

16. Chai, X. J. et al. Altered intrinsic functional brain architecture in children at familial risk of major depression. Biol. Psychiatry 80, 849-858 (2016).

17. Gaffrey, M. S., Luby, J. L., Botteron, K., Repovš, G. \& Barch, D. M. Default mode network connectivity in children with a history of preschool onset depression. J. Child Psychol. Psychiatry 53, 964-972 (2012).

18. Herringa, R. J. et al. Childhood maltreatment is associated with altered fear circuitry and increased internalizing symptoms by late adolescence. Proc. Natl. Acad. Sci. USA 110, 19119-19124 (2013).

19. Jacobs, R. H. et al. Increased coupling of intrinsic networks in remitted depressed youth predicts rumination and cognitive control. PLOS ONE 9, e104366 (2014).

20. Jacobs, R. H. et al. Decoupling of the amygdala to other salience network regions in adolescent-onset recurrent major depressive disorder. Psychol. Med. 46, 1055-1067 (2016).

21. Jenkins, L. M. et al. Integrated cross-network connectivity of amygdala, insula, and subgenual cingulate associated with facial emotion perception in healthy controls and remitted major depressive disorder. Cogn. Affect. Behav. Neurosci. 17, 1242-1254 (2017).

22. Philippi, C. L., Motzkin, J. C., Pujara, M. S. \& Koenigs, M. Subclinical depression severity is associated with distinct patterns of functional connectivity for subregions of anterior cingulate cortex. J. Psychiatr. Res. 71, 103-111 (2015).

23. Thomason, M. E. et al. Altered amygdala connectivity in urban youth exposed to trauma. Soc. Cogn. Affect. Neurosci. 10, 1460-1468 (2015).

24. Workman, C. I. et al. Subgenual cingulate-amygdala functional disconnection and vulnerability to melancholic depression. Neuropsychopharmacology 41, 2082-2090 (2016).

25. Bessette, K. L. et al. Reliability, convergent validity and time invariance of default mode network deviations in early adult major depressive disorder. Front. Psychiatry 9, 244 (2018).

26. Workman, C. I. et al. A novel resting-state functional magnetic resonance imaging signature of resilience to recurrent depression. Psychol. Med. 47, 597-607 (2017).

27. Langenecker, S. A. et al. Cognitive control neuroimaging measures differentiate between those with and without future recurrence of depression. Neuroimage Clin. 20, 1001-1009 (2018).

28. An, J. et al. Differential effects of antidepressant treatment on long-range and short-range functional connectivity strength in patients with major depressive disorder. Sci. Rep. 7, 10214 (2017).

29. Anand, A. et al. Antidepressant effect on connectivity of the mood-regulating circuit: an fmri study. Neuropsychopharmacology 30, 1334-1344 (2005).

30. Andreescu, C. et al. Resting state functional connectivity and treatment response in late-life depression. Psychiatry Res. 214, 313-321 (2013).

31. Cullen, K. R. et al. Neural correlates of antidepressant treatment response in adolescents with major depressive disorder. J. Child Adolesc. Psychopharmacol. 26, 705-712 (2016).

32. Goldstein-Piekarski, A. N. et al. Intrinsic functional connectivity predicts remission on antidepressants: a randomized controlled trial to identify clinically applicable imaging biomarkers. Transl. Psychiatry 8, 57 (2018).

33. Karim, H. T. et al. Intrinsic functional connectivity in late-life depression: trajectories over the course of pharmacotherapy in remitters and non-remitters. Mol. Psychiatry 22, 450-457 (2017).

34. Li, B. et al. A treatment-resistant default mode subnetwork in major depression. Biol. Psychiatry 74, 48-54 (2013).

35. Posner, J. et al. Antidepressants normalize the default mode network in patients with dysthymia. JAMA Psychiatry 70, 373-382 (2013).

36. Qin, J. et al. Predicting clinical responses in major depression using intrinsic functional connectivity. NeuroReport 26, 675-680 (2015).

37. Sikora, M. et al. Salience network functional connectivity predicts placebo effects in major depression. Biol. Psychiatry Cogn. Neurosci. Neuroimaging 1, 68-76 (2016).

38. Wang, L. et al. The effects of antidepressant treatment on resting-state functional brain networks in patients with major depressive disorder. Hum. Brain. Mapp. 36, 768-778 (2015).

39. Weinstein, J. J. et al. Effects of acute tryptophan depletion on raphé functional connectivity in depression. Psychiatry Res. 234, 164-171 (2015).

40. Wittchen, H.-U. \& Fydrich, T. Strukturiertes klinisches Interview für DSM-IV. Manual zum SKID-I und SKID-II (Hofgrefe, Göttingen, 1997).

41. Wakefield, J. C. \& Schmitz, M. F. When does depression become a disorder? Using recurrence rates to evaluate the validity of proposed changes in major depression diagnostic thresholds. World Psychiatry 12, 44-52 (2013).

42. Fox, M. D. et al. The human brain is intrinsically organized into dynamic, anticorrelated functional networks. Proc. Natl. Acad. Sci. USA 102, 9673-9678 (2005).

43. Sheline, Y. I., Price, J. L., Yan, Z. \& Mintun, M. A. Resting-state functional mri in depression unmasks increased connectivity between networks via the dorsal nexus. Proc. Natl. Acad. Sci. USA 107, 11020-11025 (2010).

44. Whitfield-Gabrieli, S. \& Nieto-Castanon, A. Conn: a functional connectivity toolbox for correlated and anticorrelated brain networks. Brain Connect. 2, 125-141 (2012).

45. Rush, A. J., Gullion, C. M., Basco, M. R., Jarrett, R. B. \& Trivedi, M. H. The inventory of depressive symptomatology (IDS): psychometric properties. Psychol. Med. 26, 477-486 (1996).

46. Huffziger, S. \& Kühner, C. Die Ruminationsfacetten brooding und reflection: eine psychometrische evaluation der deutschsprachigen version RSQ-10D. Z. Klin. Psychol. Psychother 41, 38-46 (2012).

47. Ochsner, K. N. \& Gross, J. J. The cognitive control of emotion. Trends Cogn. Sci. 9, 242-249 (2005).

48. Green, S. et al. The neural basis of conceptual-emotional integration and its role in major depressive disorder. Soc. Neurosci. 8, $417-433(2013)$.

49. Treynor, W., Gonzalez, R. \& Nolen-Hoeksema, S. Rumination reconsidered: a psychometric analysis. Cogn. Ther. Res. 27, 247-259 (2003).

50. Aldao, A., Nolen-Hoeksema, S. \& Schweizer, S. Emotion-regulation strategies across psychopathology: a meta-analytic review. Clin. Psychol. Rev. 30, 217-237. https://doi.org/10.1016/j.cpr.2009.11.004 (2010).

51. Varoquaux, G. Cross-validation failure: small sample sizes lead to large error bars. Neuroimage 180, 68-77 (2018).

52. Zuo, X.-N., Xu, T. \& Milham, M. P. Harnessing reliability for neuroscience research. Nat Hum Behav 3, 768-771 (2019). 
53. Faul, F., Erdfelder, E., Buchner, A. \& Lang, A.-G. Statistical power analyses using $G^{\star}$ Power 3.1: tests for correlation and regression analyses. Behav. Res. Methods 41, 1149-1160 (2009).

54. Cohen, J. Statistical power analysis for the behavioural sciences (Academic Press, San Diego, 1969).

55. Power, J. D., Barnes, K. A., Snyder, A. Z., Schlaggar, B. L. \& Petersen, S. E. Spurious but systematic correlations in functional connectivity MRI networks arise from subject motion. Neuroimage 59, 2142-2154 (2012).

56. Caballero-Gaudes, C. \& Reynolds, R. C. Methods for cleaning the bold fmri signal. Neuroimage 154, 128-149 (2017).

\section{Acknowledgements}

This study was principally funded by a Swiss National Science Foundation grant (320030L_153449/1) to QJMH and by a Deutsche Forschungsgemeinschaft grant (WA 1539/5-1) to HW. Additional funds were provided by a the Clinical Research Priority Program "Molecular Imaging" at the University of Zurich to KES and ES. KES was additionally supported by the René and Susanne Braginsky Foundation. QH additionally acknowledged support by the UCLH NIHR BRC.

\section{Author contributions}

Q.J.M.H. and H.W. conceived and designed the study with critical input by K.E.S. and E.S. I.M.B., J.W., I.S. and L.K. collected the data under the supervision of Q.J.M.H. and H.W. K.E.S. was the study sponsor in Zurich. Q.J.M.H., E.S., K.E.S. and H.W. acquired funding for the study. I.M.B. planned and performed the analyses and wrote the manuscript under supervision of Q.J.M.H. L.K. and I.M.V. gave advice on the analyses. All authors provided critical comments, read and approved the manuscript.

\section{Competing interests}

The authors declare no competing interests.

\section{Additional information}

Supplementary Information The online version contains supplementary material available at https://doi. org/10.1038/s41598-020-79170-9.

Correspondence and requests for materials should be addressed to I.M.B.

Reprints and permissions information is available at www.nature.com/reprints.

Publisher's note Springer Nature remains neutral with regard to jurisdictional claims in published maps and institutional affiliations.

(1) Open Access This article is licensed under a Creative Commons Attribution 4.0 International License, which permits use, sharing, adaptation, distribution and reproduction in any medium or format, as long as you give appropriate credit to the original author(s) and the source, provide a link to the Creative Commons licence, and indicate if changes were made. The images or other third party material in this article are included in the article's Creative Commons licence, unless indicated otherwise in a credit line to the material. If material is not included in the article's Creative Commons licence and your intended use is not permitted by statutory regulation or exceeds the permitted use, you will need to obtain permission directly from the copyright holder. To view a copy of this licence, visit http://creativecommons.org/licenses/by/4.0/.

(c) The Author(s) 2020 